Inearing the moral aspect of the quection, which wo think all will admit to be one of primary importance, we would wro that, in attending to the routine duties of medicine and eurgery for a limited period, the student is surely, though perhaps imperceptibly, picking up a great amount of lnowledge, which he cannot learn elsewhere, but which is indispensable to his future career.

If we take the lowest grade of professional duties, the pharmaceutical department, we shall find that competence for their due performance is by no means an useless or unnecessary accomplishment; for not only does the student of practical pharmacy become conversant with the best methods of combining and administering drugs, but he insensibly learns the elements of chemistry. How often have we soen inconvenient and incompatible formulø prescribed, to the great annoyance of dispensers and to the disgust of patients, from the prescribing practitioner's want of practical acquaintance with pharmaceutical manipulations.

Again, if we turn our attention to the more elevated portions of medical practice, and those in which we are daily engaged, we shall find that a knowledge of those common diseases which are most prevalent among the community can only be acquired by attending the routine of a practitioner's duties. Such diseases as measles, hoopingcough, the disorders attendant upon dentition-in fact, the whole range of infantile maladies-are rarely sean in hospitals, but are of constant occurrence in ordinary practice; and the common diseases of females, and of old age, which demand our services in a private capacity, are seldom witnessed and studied in our medical schools. The hospitals receive, as they ought to do, many of those cases of extraordinary danger and difficulty which demand the application of the highest skill and science, and which receive it at the hands of our hospital functionaries; but the students who throng the theatre when some great operation is to be performed, ought to remember that such cases will rarely occur to them in their subsequent career; and although it is perfectly just to expect from every candidate for examination that he should be prepared for the gravest emergencies of his profession, yet a great majority will gain their living by the performance of those routine duties and by attendance on such common cases as are of daily and hourly occurrence.

Another point to which we may not inaptly refer as being important in future practice, is the insight given to the student into the psychological history of human nature, while attending to "common things". We need not remind our readers, that a great part of our duties as practitioners, consists in acting upon the mental as well as the physical constitution of our patients, in raising their hopes, in calming their fears, in combating unfounded prejudices, in consoling their griefs, in allaying their anxieties, in bearing their weaknesses. Heaven forbid that we should ever attempt to gain an ascendancy, over the minds of our patients for any but honourable and Christian ends; but when guided by high principles and honest motives, there can be no doubt that such a knowledge of human nature may be turned to the best account in the treatment of disease. In hospitals and medical schools, this moral management of patients is necessarily overlooked; but in private practice it is of essential importance; and a student migy learn from a judioious private master that tact upon which will depend much of the success or failure of his future profomional lifo.

\section{ORIGITAT COMDURICAMOTS.}

\section{LECTURES DELIVERED AT THE LOCK HOSPITAL, LONDON.}

By HENRY LEE, Esq., F.R.C.S., Surgeon to the Hospital.

\section{No. VI.}

Is the examples of artificial inoculation which we have hitherto considered, suppuration of the inoculated points has been a very general and marked result. In difierent cases we have found that this action has taken a greater or less time to develope itself, but that the inflammation produced by the artificial inoculations has generally been, from the first, of the suppurative character. In these cases, 80 far as we have yet seen, no additional constitutional effects have followed the artificial inoculations. In this respect, the cases have afforded examples of the law which I drelt upon in a former lecture; viz., that when a sore from the first suppurated (whether it was induced by artificial or by natural inoculation), no constitutional symptoms would be likely to result.

Suppuration, in general, is produced with great difficulty in animals. It is not easy by any simple lesion to produce in them a discharge of well-formed pus. The readiness with which this action is induced in artificial syphilitic inoculation at once tends to support the idea that animals are susceptible of the specific influence of this morbid poison, and to indicate the mode in which nature in them eliminates the virus when artificially introduced into a part. The distinct and characteristic morbid actions to which the syphilitic poison has been shown to give rise in animals, appears to me to contradict the idea which has been maintained by some that the virus may simply remain dormant in the inoculated part (being diluted and mixed with any secretions with which it may come in contact); and that, when in this diluted state it is again reinoculated upon man, it may again resume its peculiar activity. Such an idea, I say, appears to me to be inconsistent with the distinct morbid action which is induced by artificial syphilitic inoculation of animals, and the spots of suppuration in them which are thereby induced. The pustules in the cases before recorded were found to have been formed sometimes in two and very generally in three days; and although in the last series of inoculations performed in any case, very little inflammation of any kind appears often to have been produced, yet that which did exist was of the suppurative character. This circumstance renders it extremely probable that the induration which was felt at the base of these artificial inoculations arose from the general infiltration of surrounding tissue which usually accompanies suppurative inflammation, and not upon any peculiar and specific adhesive inflammation of the inoculated part.

Attempts to induce the state called "syphilisation" have not betur"sod successfuf in France as in Italy. For although it has been found that patients gradually become less and less susceptible to the influence of the specific matter derived by successive formations of pustules on themselves from the same original source, yet it has been shown that these same patients might generally be inoculated as at the commencement, when the pus was derived from a fresh patient.

The following cases are recorded by Dr. Thiry of Brussels:

CASE. A patient, who had had a variety of primary and secondary syphilitic affections and had been subject to antisyphilitic treatment for an indurated sore, was admitted into St. Peter's Hospital on the 4th of October, 1851. Several primary ulcerations existed at this time.

Oct. 7th. Three inoculations were made upon the abdomen from one of the primary sores. In twenty-four hours, each presented the characteristic pustule and areols.

Oct. 8th. Three inoculations were made as before.

Oct. 9th. Three inoculations were made with the pus derived from the first inoculations.

Oct. 10th. Three inceulations wase made with the pow 
puleced bs the inoculations of the 7 th. Thees had not inaned in eive, and were corered by a crust, which, when rived; allowed the escape of a considerable quantity of virulent pus. The inoculations made on the 9 th had succeoded. Oot. 11th. Three fresh incculations were performed with the pus of the chancres inoculated on the 8th.

Oct. 12th. Three areolar pustules had followed the inocuintions of the previous day. The pustules produced by the incoulations of the 10th showed a tendency to fade. Under each pustule there was some thickening of the cellular tisoue, but not the least sign of specific induration.

Oct. 13th. The different inoculations, after having somewhat enlarged, faded away, and presented the starred appearance of some cicatrices.

Three inoculations were repeated on the 14th and 15th reopectively. The latter became less developed than the former.

Oet. 16th. Three inoculations were made with the pus of the chancres inoculated the day before.

Oct. 17th. The inoculations of the 16th had succeded, but in a very slight degree. Three fresh inoculations, made with the secretion of the most active sores, were followed with positive results.

The inoculations were continued on the $18 \mathrm{th}, 20 \mathrm{th}$, 21st, and 2ind.

Oct. 23rd. The last inoculations had now only produced a slight papular elevation, having no characteristic appearance.

Oct. 24th. Three fresh inoculations were made with such remains of purulent matter as could be collected from the different sores; one only of these inoculations gave rise to a papular elevation similar to those before mentioned; the other inoculations furnished no result.

Three similar inoculations on the 25th, ended in a papular elevation still less marked.

On the 26th, 27th, 28th, and 29th, all the inoculations became cicatrised without induration. Some further attempts were made to inoculate this patient from the secretions of her own sores, but in vain; nothing further was produced. But, on the lst of November, this same patient was inoculated with the matter taken from the sores of another woman, who had also been subject to this treatment by syphilisation.

Nov. Ind. The inoculation had succeeded; a vesicular pustule had appeared, surrounded by a red areola, faint, it is true, but still characteristic. This vesicular pustule contained a sero-purulent fluid, which was again inoculated, and again produced its specific effects. Another series of inoculations was now again commenced from this fresh source of infection, and the inoculations all succeeded as at the first. At length, after fifty-seren inoculations in all had boen practised, the experiment was given up.

The inoculated sores were healed as soon as possible, and the patient left the hospital in the beginning of December.

In a second case, the attempt to induce syphilisation was given up after sixty-three inoculations had been made, all of which succeeded; and in a third case, after twenty-five artificial ulcers had been produced.

Such cases furnish a sufficient refutation of the idea, that the state known or imagined as syphilisation can be, at will, artificially produced; yet have we, on the other hand, direct evidence that individuals and even nations, from artificial or natural causes, become susceptible to the infuence of the syphilitic poison in extremely different degrees: and we have conclusive testimony, as I believe, that the repeated inoculations of syphilitic matter whether by artificial or natural means, tends powerfully to produce such a modifieation of the effects of the poison.

In the first place it must, I think, be admitted that, as a rule, asphilitic matter derived from the same original source gradually loses its influence after successire inoculations. ixceptions mas occur to this as to every other rule, but as far as the evidence upon this point has hitherto gone, it tonds to prove that the poison derived from the same source sadually loses its effect when successively applied to diffinent parts of the anme patient. Bome of you had an opportunity of obearving the following case, for the note of which I am indebted to Mr. Grylls, our late housesurgeon.

CAsE. H. C., aged 21, came under treatment on the snd of Pebruary, 1854. She had then a syphilitic eruption of eleven weeks duration. There were several chancres about the inferior commissure, anus, and inner margins of both labia. The inguinal glands were slightly enlarged and indurated, and there was enlargement of the right nympha with raginal discharge.

February 4th. Several spots were inoculated on the right natis, from the angry looking sores on the margin of the arus and labia.

February 6th. Several fresh spots were inoculated on the left natis, from other angry looking sores.

February 11th. Each point inoculated produced a small pustule: several fresh inoculations were made with the pus of the original sores and of the artificial inoculations.

February 13th. Upon each last inoculated point a resicle had appeared.

February 14th. Each vesicle had become pustular.

February 16th. The left natis was now inoculated in several spots from the sores, both natural and artificial, which furnished the largest amount of secretion. Eighteen hours afterwards, the inoculated spots were found to be slightly reddened.

February 18th. Forty-four hours after the inoculation, no effect was perceptible. Several fresh spots were inoculated with the pus of the sures near the anus and from thoseartificially produced, on the inner side of the right natis.

February 23rd. The last inoculations had produced no effect. The inoculations of the llth were forming small dry scabs. The eruption had now faded.

February 24th. Several spots were inoculated over the sacrum with as much remaining secretion as could be obtained from any of the sores.

February 27th. The inoculations of the 24th had taken no effect.

February 28th. All the inoculated and other primary sores had healed, without having any induration.

This patient was considered to have recovered on the 2nd of March, having been under treatment exactly four weeks, and having during the last two weeks been apparently insusceptible of any farther inoculation by means of the secretion derived from her own sores.

It is probable that, in this case, if the matter had been taken from a fresh patient, the inoculations would have succeeded. But this experiment was not considered either necessary or justifiable. The treatment adopied consisted of the compound steel pill, and of some saline medicine at a time when there were some slight feverish symptoms.

It may be remarked, with regard to this case, that the eruption had persisted for some weeks before the patient came under observation, and that it would probably have faded in something like the same time, had she not been subject to any treatment at all.

The length of time that this patient had been diseased will also probably account for the little susceptibility she shewed to any farther infection.

The results obtained by experiments agree in this respect with those derived from the observation of the natural course of the disease. It will constantly happen, that persons habitually exposed to contagion or those who have long suffered from syphilitic disease, will at length become little liable to any fresh syphilitic influence from natural causes.

There are some who enjoy a comparative immunity from syphilitic infection. What the conditions are which confer this peculiarity have not been satisfactorily ascertained. But there can be no doubt that, while in some individuals it is found to exist naturally, that in others it may, for time at least, be acquired.

This peculiarity is found to exist, not only among individuals but also among large classes of people, and even to extend to nations. In nations, as in individuals, this comparative immunity may be natural. In both it may in 20 mo degree be acquired. Whoerer will take the trouble to look ore 
the hoepitals in France, and compare the caces there ceen with thoes treated in the hospitals in. England, will be struck rith the mildness of the symptoms in the former $28 \mathrm{com}$ pared with the latter. Yet there have been periods in the history of the disease in which the French have suffered most severely. Now, however, it appears by successive trensmission to have become so modified as scarcely to sppear to be the same affection. Some French authors, and among others MM. Maisonneuve and Montanier, have supposed that a diseased parent has transmitted the immunity which he had acquired in some measure to his offspring; and that thus, through a series of transmissions, the disease has generally become modified for the whole nation.

It must be admitted that any animal poison may become more or less active in its operation, from various causes quite independent of any peculiarity in the constitution of those affected. But a greater or less activity of the poison itself will certainly not account for the facts which have been noticed regarding syphilitic infection. It is well known that individuals exposing themselves to the same source of disease will often be very differently affected: the same thing is true with regard to nations. In the year 1812 , it was noticed by Dr. Fergusson that the British army suffered most severely during the four years that it had then been in Portugal, while the inhabitants of the country had the disease in very mild forms. They were "cured by topical remedies alone, and," says Dr. Fergusson, "I have lived long enough among them to ascertain, that their return to hospital under such circumstances for secondary symptoms is far from an universal, or even a frequent occurrence. The renereal lists amount to forty-six, and two of them only are taking mercury. None of the ulcers are such trifling cases of chancre as we have seen at home; nor even such as an English soldier would run with in affright to seek the succour of his surgeon. To these, the Portuguese soldier pays no attention whatever; he does not consider them to be a hindrance to him in any wanner, and I have seen him turn out for duty with ulcers that made me shudder to look at, though both he and his medical attendant considered them as nothing."

While the native soldiers were thus treated by topical remedies alone, and seldom had an y constitutional symptoms, the British often "sustained the most melancholy of all mutilations," and the venereal ulcerations in them were not only "found more intractable to the operation of mercury than under similar circumstances at home"; but the constitution, while under the influence of the remedy, became affected with the secondary symptoms in a proportion that could not have been expected. Such were the very different results in the two classes of persons infected from the same sources. In the one, whose constitutions appeared to have become familiarised with the disease, it produced, as a rule, only its local effects; in the other, who did not enjoy the same unenviable privilege, not only were the local affections more severe, but the system became often affected, and that in spite of appropriate medical treatment.

From such considerations, it will at once appear how fallacious any conclusions are likely to be that are derived exclusively from the observation of patients who have long been subject to disease either in its direct or hereditary forms. Any data derived from venereal hospitals, unless care be taken in the selection of the cases, may from this cause prove utterly valueless when applied to cases met with in private practice; and especially are the facts furnished by any particular class of persons liable to mislead, if inadrertently applied to another class, placed in a different position. Thus, from observations made in the army, Mr. Rose, in the year 1817, published some observations on the treatment of syphilis, with an account of ceveral cases of that disease in which a cure was effected without mercury; and, from his experience among soldiers, he was led to believe that he might adopt the ame means of treatment in private practice. But we have it recorded upon the authority of $\operatorname{Sir}$ B. Brodie, that here it was uneuccesaful, and that $\mathbf{M r}$. Bose was at last induced again to bave recourse to morevry in the treatment of spphilitic affections in private. Bince Mr. Roep's publiention, mans other army surgeons have adopted the non-mencesict plan of treatment, and have continued it up to tho preacen time. This they certainly would not have done, had they not found it successful. But it must be remembered that the cases which they have to do with occur in thowe who are often in some measure syphilised. The patients whom they see have comparatively rarely the disease for the first time; their systems have in some measure become accustomed to the influence of the poison, and the forms both of primary and secondary disease in them are most materially modified thereby. A person who has repeatediy contracted primary syphilis is in some measure pleced under the same circumstances as a patient who has been repeatedly inoculated artificially; and we possess sufficient evidence, derived both from observation and experiment, to show that, under such circumstances, the local disease is altered in its characters, and that no additional constitutional affection is likely to be induced.

The modification of the action of the syphilitic poison, in consequence of repeated inoculation, although it has of late years assumed a new name, is not, as we have already seen, a new subject. The conclusion to which Dr. Fergueson arrived in the year 1812 was, that the disease had become so much mitigated in Portugal, by reason of general diffnsion or other causes, that, after running a mild course, it exhausted itself, and ceased spontaneously. Dr. Fergusson further mentions, that at that time, he had reason to believe that in other countries the disease became modified in a similar way; that in certain German regiments, and in some districts of the Russian empire, the medical attendants had found that mercury was not necessary for the treatment of syphilis; and that, in the patients to whom he referred, the disease, from being allowed to run its course probably for ages, had become as weak as it was found to be in the Portuguese.

The conclusion arrived at by Dr. Fergusson is remarkable, as furnishing a mcans of accounting for what he had himself observed, and as having been revived and adopted to account for the more extended series of observations and experiments of the present day.

All adventitious diseases, he says, that are not connate, endemic, and sporadic, appear more or less to run this course of exhausting themselves while retained upon the same ground to which they have been transplanted. But let the field be changed, and fresh sources of development be presented, they instantly resume their primary powers, and, taking a fresh departure of violence, repeat the almost forgotten inflictions of their original visitation. The powers which they thus acquire bear some resemblance to a phenomenon which is every where observable in the vegetable kingdom. The same species of seed may be sown upon the same ground until it shall so degenerate in point of vigour as to become almost incapable of reproducing itself; but let it be changed to any other of any kind, though even of far inferior quality, it will immediately display new powers of life, and fructify and vegetate with its native strength: Similar to the above appears to be the inoculation of the exhausted syphilitici virus of Portugal (though evidently the same disease) into the constitution of the British or other stranger. It is in some measure new, therefore unfriendly; and seems to have the power of exciting new actions of more than ordinary violence.

The Portuguese, through apathy, and at a dreadful price levied on the generations that are passed, and never, in all probability, to be redeemed by their descendants, appear to have gained a great exemption from both syphilis and variola ; but the price is too high for us ever to offer up our bodies to be the unresisting subjects of disesse, the fatal consequences of which, though they might go far to extinguish one or two ills, would be felt in the deterioration of our race to the most distant ages. 